\title{
Morphological and immunocytochemical analysis of Escherichia coli-specific surface antigens in wildtype strains and in recombinant Vibrio cholerae
}

\author{
Stefan Lüdi · Joachim Frey · Didier Favre · Jean-François Viret • \\ Verena Ziethlow $\cdot$ Kathrin Kühni Boghenbor $\cdot$ Michael H. Stoffel
}

Received: 1 May 2007/Accepted: 19 July 2007/Published online: 21 August 2007

(C) Springer Science+Business Media B.V. 2007

\begin{abstract}
Adhesion is the first step in the pathogenesis of enterotoxigenic Escherichia coli infections. The genes encoding the most prevalent adhesion factors CFA/I, CS3 and CS6 were cloned into Vibrio cholerae strain CVD $103-\mathrm{HgR}$ and expression of fimbriae was investigated in wildtype and recombinant strains by transmission electron microscopy in conjunction with immunolabelling and negative staining. Negative staining was effective in revealing CFA/I and CS3, but not CS6. Although morphology of fimbriae differed between wildtype and recombinant strains, corresponding surface antigens were recognized by specific antibodies. The present study provides evidence that ETEC-specific fimbriae can adequately be expressed in an attenuated $V$. cholerae vaccine strain and that immunoelectron microscopy is a critical tool to validate the surface
\end{abstract}

S. Lüdi · V. Ziethlow · K. K. Boghenbor ·

M. H. Stoffel ( $\square)$

Division of Veterinary Anatomy, Vetsuisse Faculty

University of Berne, P.O. Box 8466, 3001 Bern,

Switzerland

e-mail: michael.stoffel@ita.unibe.ch

S. Lüdi · J. Frey

Institute of Veterinary Bacteriology, Vetsuisse Faculty

University of Berne, P.O. Box 8466, 3001 Bern,

Switzerland

D. Favre - J.-F. Viret

Berna Biotech Ltd, Rehhagstrasse 79, Bern 3018,

Switzerland expression of antigens in view of their possible suitability for recombinant vaccines.

Keywords Colonization factor $\cdot \mathrm{CFA} / \mathrm{I}$.

CS3 - CS6 - Vaccine .

Transmission electron microscopy

\section{Introduction}

Infection with enterotoxigenic Escherichia coli (ETEC) is one of the major causes of diarrhea affecting travelers and residents in endemic countries (Jiang et al. 2002; Oyofo et al. 2001). A number of antibiotic drugs are able to prevent diarrhea for a limited period of time, but the potential for adverse effects and the risk of selecting resistant pathogens calls for a restrictive use of antibiotics and for other prophylactic measures. Therefore an enormous effort has been initiated worldwide to develop a safe and effective vaccine against ETEC.

Infections with ETEC require proper adhesion of bacteria to the intestinal mucosa. This first step in the pathogenesis of ETEC diarrhea is mediated through specific surface structures called pili or fimbriae (Jonson et al. 2005), which allow the bacteria to colonize the mucosal surface of the small intestine. Termed Colonization Factors (CF), these proteins are subdivided into colonization factor antigens (CFA), which include CFA/I, CFA/II, CFA/III and CFA/IV, and a number of so-called coli surface antigens (CS). 
CFA/I and CFA/III are rodlike fimbrial antigens with diameters of 6-8 nm (Knutton et al. 1985; Levine et al. 1984). CFA/II itself consists of three distinct coli surface (CS) antigens, CS1, CS2 and CS3. Whereas CS1 and CS2 are morphologically similar to CFA/I, CS3 was shown to consist of fine fibrils with a diameter of 2-3 nm (Levine et al. 1984) CFA/IV comprises three distinct coli surface antigens, CS4, CS5 and CS6. CS4 and CS5 are rodlike fimbriae, whereas the morphology of CS6 has not yet been elucidated (de Lorimier et al. 2003; Knutton et al. 1989; Nishikawa et al. 1998).

In order to assess the potential of $V$. cholerae as a carrier, the genes encoding the most common ETEC surface antigens, CFA/I, CS3 and CS6, were cloned into the vaccine strain CVD 103-HgR (Ketley et al. 1993) alone or in combination. Using transmission electron microscopy (TEM) in conjunction with negative staining and immunolabelling, recombinant CVD strains were then compared to ETEC wildtype strains. The present study provides evidence that specific surface antigens can be expressed at the surface of the $V$. cholerae CVD $103-\mathrm{HgR}$ and that resulting recombinant strains may be expected to elicit a proper antibody response.

\section{Materials and methods}

Bacterial strains, culture conditions and preparation of whole mounts for transmission electron microscopy (TEM)

ETEC wildtype and recombinant $V$. cholerae strains expressing the respective surface antigens as well as the plasmids used have been reported previously (Favre et al. 2006). Stability of plasmids in recombinant strains was analyzed by growing the cultures in exponential phase for ten generations without selective pressure of antibiotics. Persistence of plasmids was then confirmed in single colony isolates by growth on solid medium containing the corresponding antibiotics and by plasmid purification.

Bacteria were grown on solid CF-medium (Evans et al. 1979) overnight at $37^{\circ} \mathrm{C}$. Antibiotics were included as appropriate $(34 \mu \mathrm{g} / \mathrm{ml}$ of Chloramphenicol, $50 \mu \mathrm{g} / \mathrm{ml}$ of Kanamycin and $50 \mu \mathrm{g} / \mathrm{ml}$ of
Ampicillin, either alone or in combination). Thereafter, bacteria were rinsed off the culture plates with $0.85 \% \mathrm{NaCl}$ pre-warmed to $37^{\circ} \mathrm{C}$ and the bacterial suspension was fixed immediately by adding an equal volume of paraformaldehyde fixative (final concentration: $0.1 \%$ paraformaldehyde in $0.01 \mathrm{M}$ phosphatebuffered saline, $\mathrm{pH}$ 7.4) or of diluted Karnovsky's fixative (final concentration: $2.5 \%$ glutaraldehyde, $2 \%$ paraformaldehyde in $0.1 \mathrm{M}$ cacodylate buffer, $\mathrm{pH} 7.4$ ), respectively. Bacteria were then adsorbed onto parlodion-coated copper or nickel grids by placing a grid upside down onto a drop of bacterial suspension. Adsorbed bacteria were washed on a drop of $\mathrm{H}_{2} \mathrm{O}$ for $5 \mathrm{~min}$.

\section{Negative staining}

Negative staining was performed by floating the grids on a drop of $0.25 \%$ phosphotungstic acid (PTA) with $0.01 \%$ bovine serum albumin in $\mathrm{H}_{2} \mathrm{O}$ for 30-40 s. Excess liquid was blotted off with filter paper and samples were air dried.

\section{Immunolabelling}

In order to prevent non-specific binding of primary antibodies, samples were initially blocked for $20 \mathrm{~min}$ with phosphate-buffered saline containing $0.2 \%$ bovine serum albumin and $0.05 \%$ Tween 20 (PBSBT). As this step was shown to be unnecessary to prevent background labelling, it was omitted in later experiments. Antibodies used included mouse monoclonal anti-CFA/I and anti-CS3 as well as rabbit polyclonal anti-CS3 and anti-CS6 antibodies. All the antibodies were raised against purified antigens (provided by Dr. F. Cassels, NIH, Bethesda, Maryland, USA). Rabbit sera were pre-adsorbed against a panel of bacterial strains in order to remove irrelevant antibodies. Monospecificity of all antibodies used was confirmed on Western blots as described (Burnette 1981).

For immunogold labelling, TEM grids were floated on corresponding primary antibodies for $60 \mathrm{~min}$ (diluted 1:100 or 1:50 in PBS-BT). The grids were washed in PBS-BT for 5 min and incubated for another $60 \mathrm{~min}$ on a drop of either goat $15 \mathrm{~nm}$ gold-conjugated 
anti-mouse $\mathrm{IgG}$ diluted $1: 100$ in PBS-BT or goat $15 \mathrm{~nm}$ gold-conjugated anti-rabbit IgG (both from British Biocell International, Brunschwig, Germany).

Double labelling was performed by incubation of grids with a mixture of primary antibodies (mouse monoclonal anti-CFA/I and rabbit polyclonal antiCS3 antibodies) followed by incubation with a mixture of both appropriate secondary antibodies, i.e., goat $10 \mathrm{~nm}$ gold-conjugated anti-mouse $\mathrm{IgG}$ (Sigma, Missouri, USA) and goat $15 \mathrm{~nm}$ gold-conjugated anti-rabbit IgG (British Biocell International, Brunschwig, Germany). Finally, the grids were washed twice in $\mathrm{H}_{2} \mathrm{O}$ for 5 min. After blotting off excess liquid with filter paper, negative staining and air drying, grids were examined in a Zeiss 109 transmission electron microscope (Zeiss, Oberkochen, Germany) equipped with a GATAN wide angle slow scan CCD Camera 689 (GATAN GmbH, Munich, Germany).
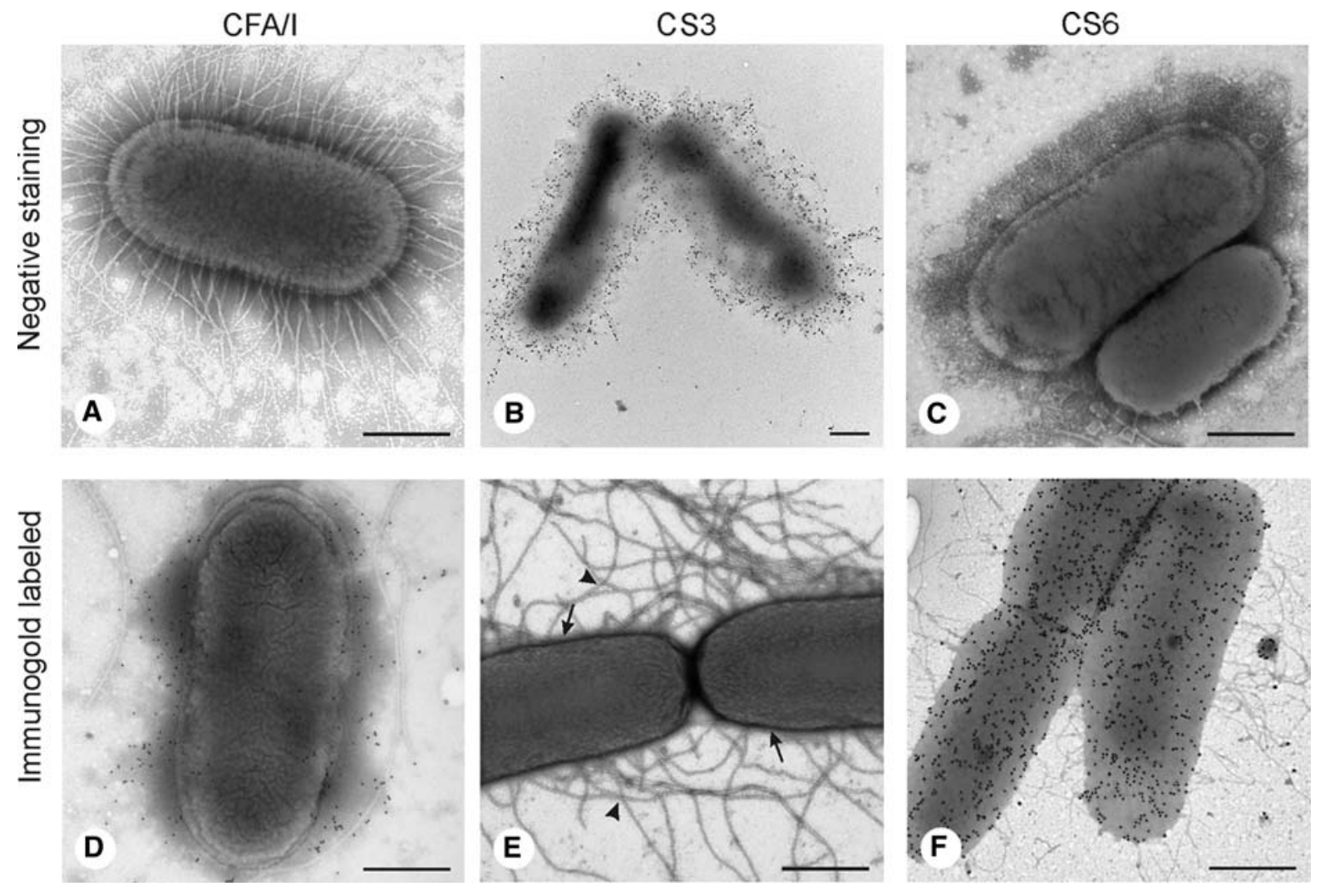

Fig. 1 Expression of CFA/I antigen in wildtype strain H10407 $(\mathbf{A}, \mathbf{B})$, of CS3 antigen in wildtype strain DS7-3 (C, D) and of CS6 antigen in wildtype strain E34420A (E, F) as revealed by negative staining and immunogold labelling. Surface expression of CS6 fimbriae is combined with CFA/III fimbriae in

\section{Control experiments}

Control experiments included negative staining and immunolabelling of host strain CVD 103-HgR with antiCFA/I, anti-CS3 and anti-CS6 antibodies. Specificity of labelling was assessed by simply omitting the primary antibodies, as well as by using a rabbit anti-calcitonin antibody (Anawa Biomedical Services and Products, Zürich, Switzerland) and a mouse anti-osteoprotegerin antibody (R\&D Systems, Oxon, UK) as irrelevant substitutes for pertinent primary antibodies.

\section{Results}

Expression of CFA/I, CS3 and CS6 fimbriae in wildtype strains

All antibodies used identified a single protein band of the appropriate molecular weight in Western blot 
analysis (data not shown). Analysis of plasmid persistence revealed that plasmid loss was below $1 \%$ in the absence of antibiotic selection.

Expression of CFA/I was examined in the two CFA/ I-positive ETEC wildtype strains H10407 (Fig. 1A, B) and CD79a (not shown). Negative staining clearly revealed long, spiny and rod-like fimbriae (Fig. 1A). The identity of these fimbriae as CFA/I was corroborated by immunogold labelling (Fig. 1B).

ETEC strains DS7-3 (Fig. 1C, D) and DS198-1 (not shown) were used as reference strains for the CS3 surface antigen. In contrast to CFA/I, CS3 appeared to be thin and curly fibrils, as shown by negative staining (Fig. 1C). Likewise, immunogold labelling with both monoclonal and polyclonal antibodies was highly specific and, thus, unequivocally confirmed these hair-like structures to be CS3 surface antigens (Fig. 1D). CFA/I and CS3 fimbriae expression requires the presence of one regulator gene. CsfR and rns are fully interchangeable regulators and one or the other was used at different periods in the course of the experimental work leading to the present publication.

As for CS6, negative staining only provided evidence of a narrow margin of phosphotungstic acid surrounding the bacteria. Consequently, only one type of surface structure was detected in the bivalent wildtype strains examined, i.e., long wavy CFA/III fimbriae in E34420A (Fig. 1E) and rigid but long CS5 fimbriae in E17018A (not shown). However, immunogold labelling with polyclonal CS6-specific antibodies yielded evidence of CS6 fimbriae surface expression in both strains, although labelling density was much higher in E34420A (Fig. 1F) than in E17018A (not shown). Interestingly, labelling of CS6 structures was superimposed over the cell body in both instances (Fig. 1F) and, as opposed to CFA/I and CS3, no halo of labelled fimbriae surrounding the cell body was seen.

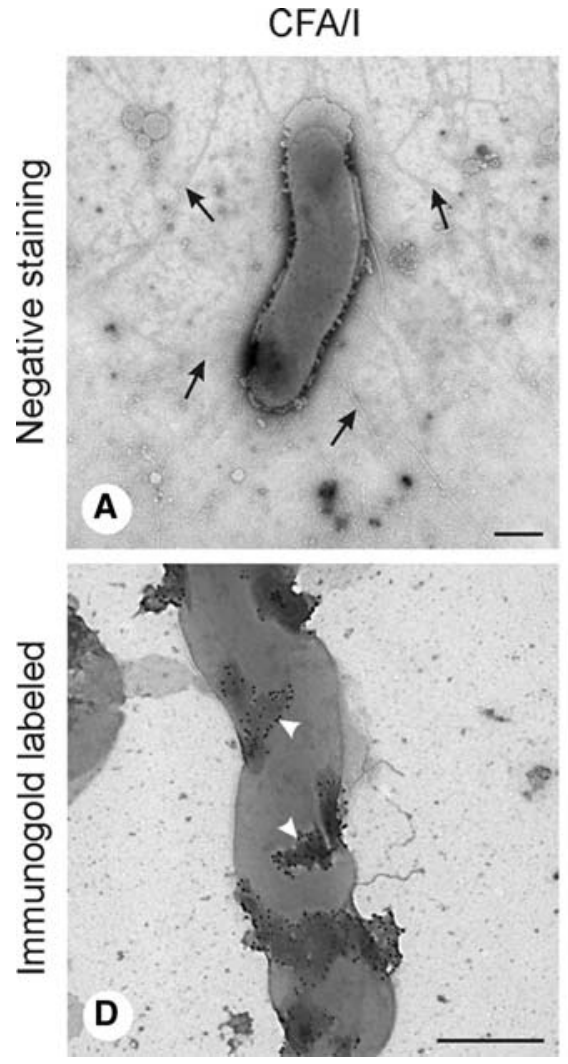

Fig. 2 Expression of CFA/I, CS3 and CS6 antigens in monovalent recombinant strains as revealed by negative staining (A, C, E) and immunogold labelling (B, D, F).
CS3
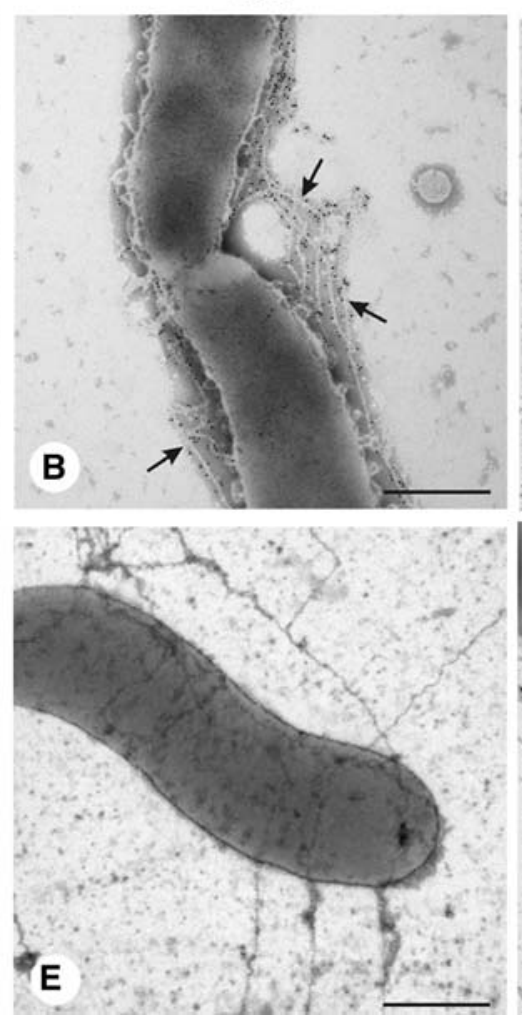

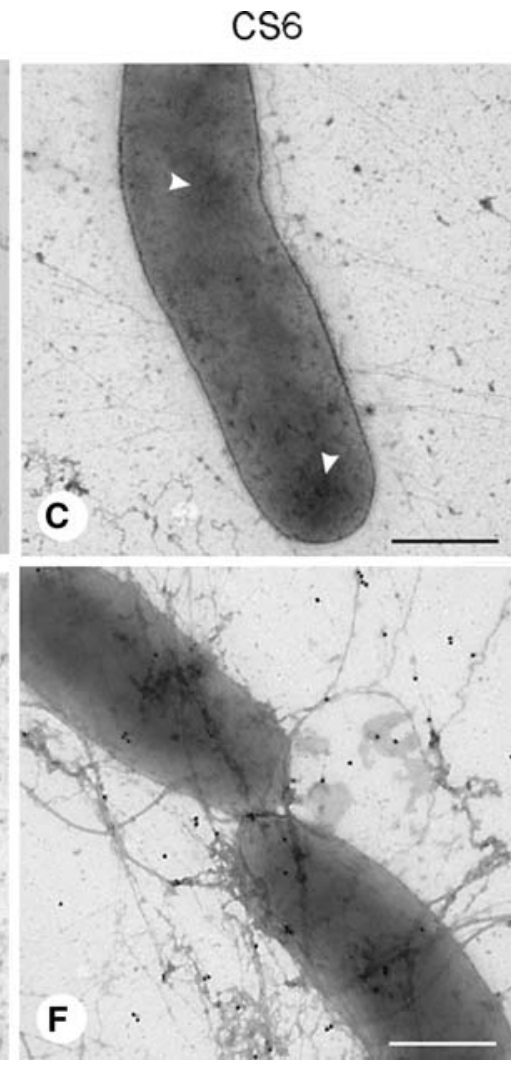

CFA/I (arrows) is displayed by BB01 (A, B), CS3 (arrowheads) by CVD 103-HgR(pSSVI215-CS3/pM392) (C, D) and CS6 by CVD 103-HgR(pM295) (E, F). Scale bar $=0.5 \mu \mathrm{m}$ 
Expression of CFA/I, CS3 and CS6 in recombinant monovalent $V$. cholerae strains

Negative staining of strain BB01, the recombinant $V$. cholerae strain CVD 103-HgR harboring chromosomally integrated genes for CFA/I, revealed efficient expression of CFA/I fimbriae (Fig. 2A). However, these surface structures were morphologically different from their equivalents in
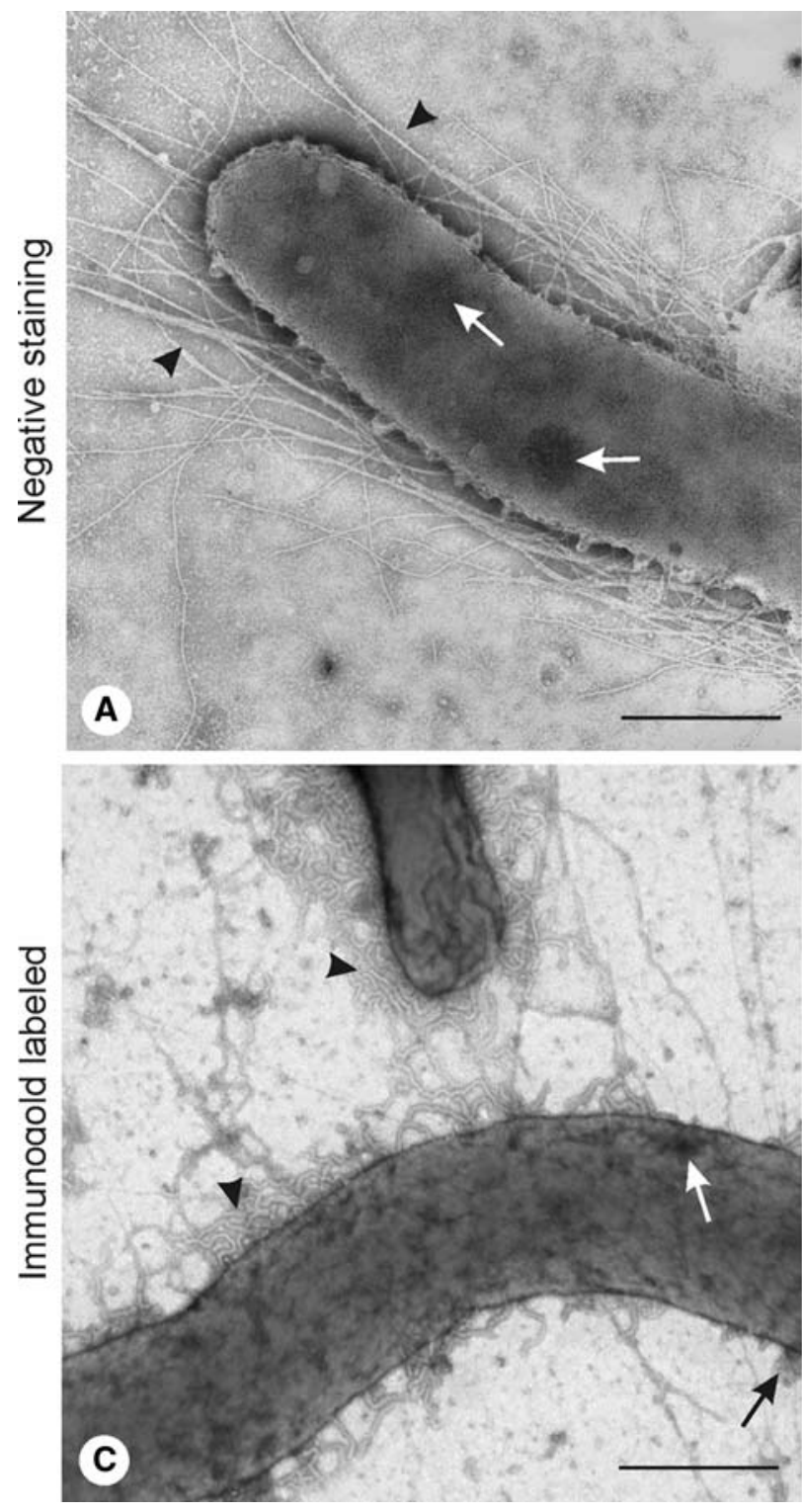

Fig. 3 Expression of CFA/I (arrowheads) and CS3 (arrows) antigens in bivalent recombinant strains CHCS3-R2 (pSSVI215-cfaI) (A, B) and BB06 (C, D) as revealed by negative staining $(\mathbf{A}, \mathbf{C})$ and immunogold labelling (B, D). wildtype strains $\mathrm{H} 10407$ and CD79a (Fig. 1A). Whereas CFA/I fimbriae in wildtype ETEC strains were stiff, rod-like and short structures, fimbriae in recombinant $V$. cholerae appeared to be longer, less densely packed, undulated and less rigid. However, immunogold labelling provided compelling evidence that these surface structures are immunogenically identical to CFA/I fimbriae (Fig. 2B).
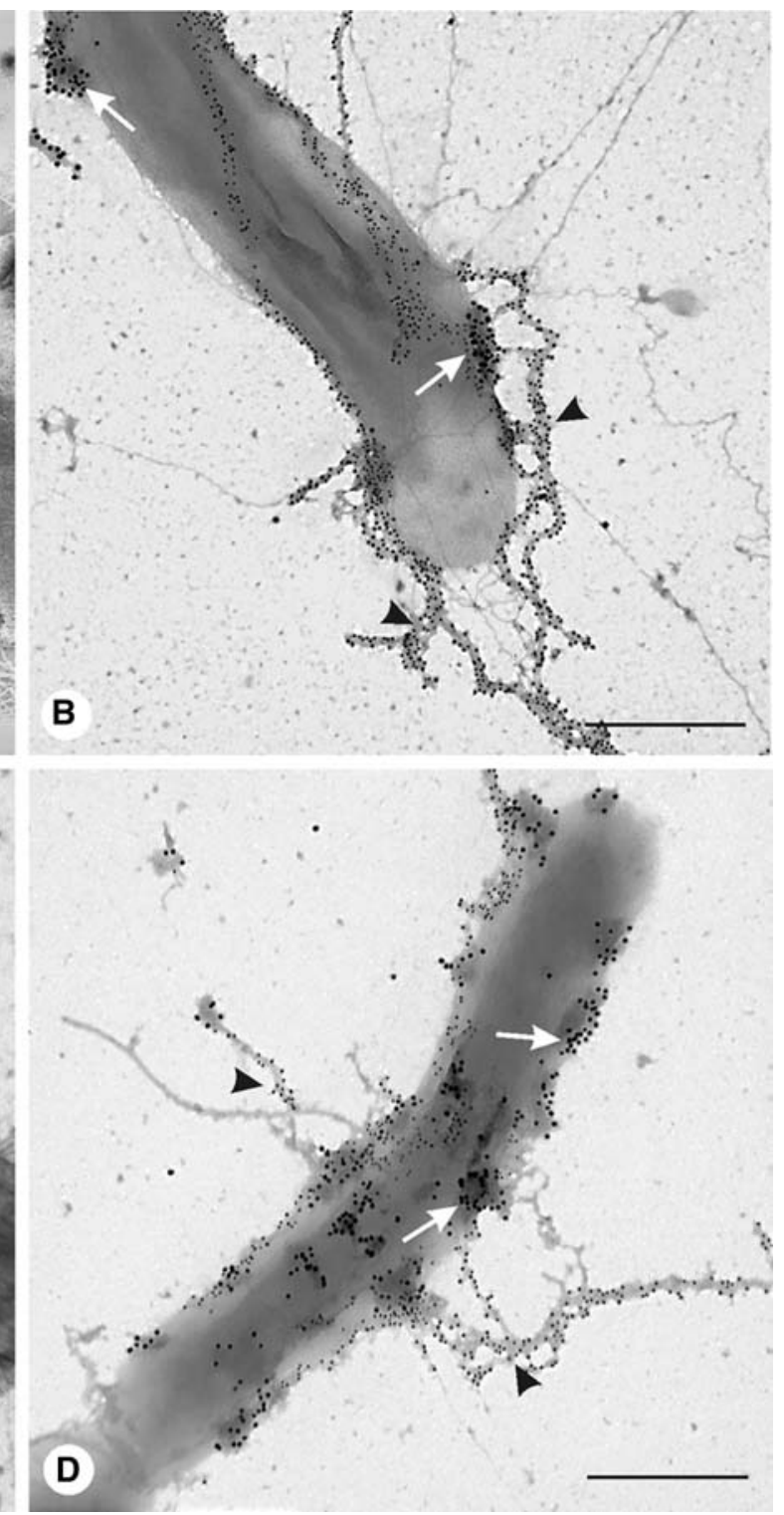

Binding sites for CFA/I are demonstrated with $10 \mathrm{~nm}$ colloidal gold (B, D; arrowheads) and binding sites for CS3 are demonstrated with $15 \mathrm{~nm}$ colloidal gold (B, D; arrows). Scale bar $=0.5 \mu \mathrm{m}$ 
In contrast to their presence in wildtype strains, CS3 fimbriae were difficult to visualize by means of negative staining in the recombinant $V$. cholerae strain CVD 103-HgR (pSSVI215-CS3/pM392) (Fig. 2C). However, their presence as wispy structures was demonstrated by immunolabelling (Fig. 2D). CS3 was expressed as bushy patches being disseminated over the entire bacterial surface. This was in contrast to CS3 expression in wildtype ETEC strains where the fimbriae uniformely covered the whole cell surface. Obviously, expression of corresponding genes produces fimbriae that are much frailer than in wildtype ETEC strains. As judged from their scattered occurrence away from bacteria, they also seem to be more easily lost during sample handling (not shown).

In accordance with the observations made on wildtype strains, CS6 could not be demonstrated morphologically on recombinant $V$. cholerae strain CVD 103-HgR (pM295) either (Fig. 2E). Notwithstanding, the specific labelling signal obtained with polyclonal rabbit anti-CS6 antibodies (Fig. 2F) was similar to the labelling density seen in wildtype strain E17018A (not shown).

Expression of CFA/I and CS3 in recombinant bivalent strains

On bivalent $V$. cholerae strain CHCS3-R2 (pSSVI215cfaI) (Fig. 3A), negative staining revealed CFA/I fimbriae as long and curved tubes, whereas CS3 fimbriae could only be perceived as darker lumps on the bacterial body. Double labelling (Fig. 3B) clearly revealed the presence of both CS3 and CFA/I fimbriae.

As compared to CHCS3-R2 (pSSVI215-cfaI) (Fig. 3A), presumptive CFA/I fimbriae on bivalent strain BB06 were noted as partly adhering to the cell surface and partly extending away from the bacteria (Fig. 3C). Whereas CS3 fimbriae could only faintly be sensed after negative staining, they were clearly outlined as multifocal spots by immunolabelling (Fig. 3D).

Negative controls

All host strains were devoid of background labelling when incubated with anti-CFA/I, anti-CS6 or antiCS3 antibodies. Substitution of specific primary antibodies with irrelevant counterparts abolished any labelling in wildtype strains.

\section{Discussion}

There are different strategies to create vaccines against bacterial infectious diseases, including purified antigens, inactivated whole cells, live attenuated cells of the pathogen of interest or the use of live bacterial carriers expressing the gene(s) coding for the antigen(s) of interest. Advantages of the latter approach are the availability of innocuous carrier strains, the presentation of antigens that allow the colonization process to be mimicked and the potential of inducing excellent immune responses. An important issue in this context is the need to express the antigen in its immunogenic form. Structural modifications of the antigen as compared to the wildtype strain may be associated with dissimilar epitope conformations, thus leading to suboptimal immune responses.

The goal of the present study was to investigate the potential of expressing E. coli-specific surface antigens in recombinant $V$. cholerae vaccine strains and to compare their surface expression to ETEC wildtype strains. Transmission electron microscopy, in combination with negative staining and immunogold labelling, proved itself to be an essential tool for assessment of the expression of $E$. coli-specific surface antigens in the recombinant strain CVD 103-HgR.

Culture conditions and handling protocols first had to be tailored carefully so as to ensure optimal gene expression and preservation of the delicate fimbrial structures. A number of factors, such as the composition of the culture media and washing solutions or the incubation time and temperature, all affected their presence. Centrifugation in particular turned out to be detrimental to the preservation of small fimbrial-type structures such as CS3 and CS6 (Novotny et al. 1969). Preliminary experiments thus led to the protocols reported herein.

Furthermore, phase variation has been reported in ETEC (Knutton et al. 1987; Sohanpal et al. 2001). Although this phenomenon is mainly associated with type 1 fimbriae, it should be considered as a possible explanation for the observation that CS3 fimbriae occasionally escaped morphological detection on wildtype E. coli strain DS7-3. 
Morphological analysis of wildtype ETEC strains and recombinant vaccine strains by means of negative staining provided impressive evidence of substantial differences in the surface expression of genetically and immunogenically identical CFs. Thus, manifestation of both CFA/I and CS3 genes results in morphologically clearly different structures depending on whether expression takes place in wildtype or recombinant strains. The reasons for this variability have not been elucidated to date, but it seems likely that genes other than the ones encoding for the actual fimbriae are involved in the expression process. $V$. cholerae naturally expresses Tcp fimbriae (Ehara et al. 1987; Hall et al. 1988) and the expression and secretion machinery driving the synthesis of Tcp fimbriae may affect the synthesis of some of the ETEC CFs. This possibility is particularly compelling in the case of CFA/I fimbriae in $V$. cholerae, which bear a certain resemblance to Tcp fimbriae, while being strikingly different from CFA/I fimbriae in ETEC. In the case of CS3, expression in $V$. cholerae results in a patchy pattern, whereas CS3 fimbriae seem to be synthesized all over the surface of ETEC wildtype strains. This is indicative of heterogeneity in the surface composition of $V$. cholerae as to its capacity to synthesize CS3 fimbriae. It will be interesting to investigate whether such heterogeneity correlates with some other functional properties of $V$. cholerae. However, although the appearance of the surface antigens may vary, their antigenicity is obviously preserved. Thus, CFA/I, CS3, and CS6 pilins, the structural proteins making up the CFs, were equally recognized by poly- and monoclonal antibodies in Western blots of wildtype and recombinant strains and the corresponding polypeptides were of the same size (Favre et al. 2006). In addition, wildtype and recombinant strains (both monovalent and bivalent) were specifically labelled by the same antisera. Pertaining to the latter consideration, double-labelling experiments provided evidence of coexpression of CFA/I with CS3. This aspect is essential in view of adopting a multivalent recombinant approach towards a vaccine against ETEC.

Although visualizing CS6 fimbriae has not been achieved to date (de Lorimier et al. 2003; Nishikawa et al. 1998), we made numerous attempts to provide morphological data on these surface structures in a number of strains, including wildtype strains E34420A, E17018A and B7A (data not shown), as well as the recombinant strain CVD $103-\mathrm{HgR}$ (pM295) containing the genes encoding CS6 fimbriae. Whereas CS6 fimbriae eluded morphological depiction, we were able to demonstrate their presence by immunolabelling. Interestingly, colloidal gold tags were strictly superimposed over the cell body, thus providing suggestive evidence that CS6 fimbriae are so short and thin that they do not protrude enough from the bacterial surface to become apparent after negative staining. This contention is further corroborated by the observation that, in contrast to naked bacteria, the contour of CS6-positive bacteria is highlighted by a narrow margin of retained phosphotungstic acid.

Altering the adhesion behavior of the vaccine strain CVD 103-HgR raises the question of possible changes in its virulence. Virulence of $V$. cholerae is specifically mediated by toxins. As the catalytic subunit has been removed from CVD 103-HgR, this host strain is devoid of virulence factors. Moreover, ETEC fimbriae lack any intrinsic virulence and, thus, it seems extremely unlikely that the expression of ETEC fimbriae would restore some kind of virulence in the host strain. This makes CVD 103-HgR a suitable vector. Its innocuousness and its efficacy in eliciting an immune response against ETEC-specific antigens, however, will require adequate clinical trials in vitro and in vivo.

\section{Conclusions}

Taken together, the results of the present study clearly demonstrate that single or combined expression of genes encoding colonization factors in a recombinant $V$. cholerae vaccine strain is feasible. Although the morphology of the resulting CFs may differ to some extent from their appearance in wildtype strains, CFs are readily identified by specific antibodies and, thus, obviously retain their antigenicity. Therefore, this approach holds a vast potential for the generation of customized vaccines.

Acknowledgments The technical support of Cynthia Furer, Yvonne Schlatter, Corinne Hug and Simon König is gratefully acknowledged. We also acknowledge M. Wolf, Walter Reed Army Hospital, Maryland, USA for generously providing pM295 and pM392 and Fred Cassels, NIH, Bethesda, Maryland, USA for providing purified antigens. Supported by the Federal Office for Professional Education and Technology OPET (Grant KTI 7304.1 LSPP-LS). 


\section{References}

Burnette WN (1981) "Western blotting": electrophoretic transfer of proteins from sodium dodecyl sulfate-polyacrylamide gels to unmodified nitrocellulose and radiographic detection with antibody and radioiodinated protein A. Anal Biochem 112:195-203

de Lorimier AJ, Byrd W, Hall ER et al (2003) Murine antibody response to intranasally administered enterotoxigenic Escherichia coli colonization factor CS6. Vaccine 21:2548-2555

Ehara M, Ishibashi M, Ichinose Y et al (1987) Purification and partial characterization of fimbriae of Vibrio cholerae $\mathrm{O} 1$. Vaccine 5:283-288

Evans DG, Evans DJ Jr, Clegg S et al (1979) Purification and characterization of the CFA/I antigen of enterotoxigenic Escherichia coli. Infect Immun 25:738-748

Favre D, Lüdi S, Stoffel M et al (2006) Expression of enterotoxigenic Escherichia coli colonization factors in Vibrio cholerae. Vaccine 24:4354-4368

Hall RH, Vial PA, Kaper JB et al (1988) Morphological studies on fimbriae expressed by Vibrio cholerae 01. Microb Pathog 4:257-265

Jiang ZD, Lowe B, Verenkar MP et al (2002) Prevalence of enteric pathogens among international travelers with diarrhea acquired in Kenya (Mombasa), India (Goa), or Jamaica (Montego Bay). J Infect Dis 185:497-502

Jonson AB, Normark S, Rhen M (2005) Fimbriae, pili, flagella and bacterial virulence. Contrib Microbiol 12:67-89

Ketley JM, Michalski J, Galen J et al (1993) Construction of genetically marked Vibrio cholerae O1 vaccine strains. FEMS Microbiol Lett 111:15-21
Knutton S, Lloyd DR, Candy DC et al (1985) Adhesion of enterotoxigenic Escherichia coli to human small intestinal enterocytes. Infect Immun 48:824-831

Knutton S, Lloyd DR, McNeish AS (1987) Identification of a new fimbrial structure in enterotoxigenic Escherichia coli (ETEC) serotype O148:H28 which adheres to human intestinal mucosa: a potentially new human ETEC colonization factor. Infect Immun 55:86-92

Knutton S, McConnell MM, Rowe B et al (1989) Adhesion and ultrastructural properties of human enterotoxigenic Escherichia coli producing colonization factor antigens III and IV. Infect Immun 57:3364-3371

Levine MM, Ristaino P, Marley G et al (1984) Coli surface antigens 1 and 3 of colonization factor antigen II-positive enterotoxigenic Escherichia coli: morphology, purification, and immune responses in humans. Infect Immun 44:409-420

Nishikawa Y, Helander A, Ogasawara J et al (1998) Epidemiology and properties of heat-stable enterotoxinproducing Escherichia coli serotype O169:H41. Epidemiol Infect 121:31-42

Novotny C, Carnahan J, Brinton CC Jr (1969) Mechanical removal of $\mathrm{F}$ pili, type I pili, and flagella from $\mathrm{Hfr}$ and RTF donor cells and the kinetics of their reappearance. J Bacteriol 98:1294-1306

Oyofo BA, Subekti DS, Svennerholm AM et al (2001) Toxins and colonization factor antigens of enterotoxigenic Escherichia coli among residents of Jakarta, Indonesia. Am J Trop Med Hyg 65:120-124

Sohanpal BK, Kulasekara HD, Bonnen A et al (2001) Orientational control of fimE expression in Escherichia coli. Mol Microbiol 42:483-494 